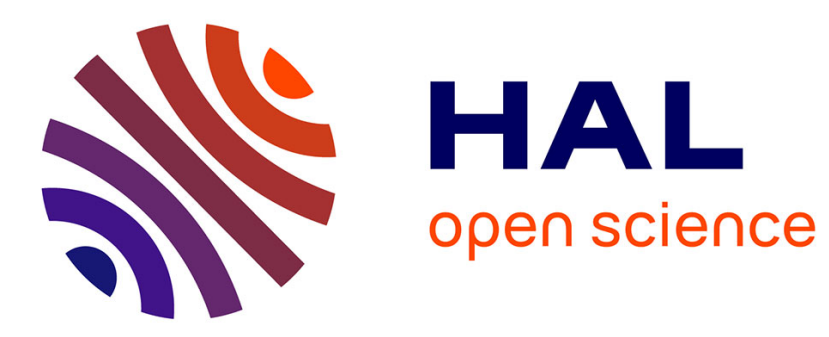

\title{
Pulling on a Filament
}

Armand Jülicher, Anthony Maggs

\section{To cite this version:}

Armand Jülicher, Anthony Maggs. Pulling on a Filament. Journal de Physique I, 1997, 7 (7), pp.823826. 10.1051/jp1:1997203. jpa-00247365

\section{HAL Id: jpa-00247365 https://hal.science/jpa-00247365}

Submitted on 1 Jan 1997

HAL is a multi-disciplinary open access archive for the deposit and dissemination of scientific research documents, whether they are published or not. The documents may come from teaching and research institutions in France or abroad, or from public or private research centers.
L'archive ouverte pluridisciplinaire HAL, est destinée au dépôt et à la diffusion de documents scientifiques de niveau recherche, publiés ou non, émanant des établissements d'enseignement et de recherche français ou étrangers, des laboratoires publics ou privés. 


\title{
Short Communication \\ Pulling on a Filament
}

\author{
Armand Ajdari $\left({ }^{1, *}\right)$, Frank Jülicher $\left({ }^{2}\right)$ and Anthony Maggs $\left({ }^{1}\right)$ \\ $\left({ }^{1}\right)$ Laboratoire de Physico-Chimie Théorique $\left({ }^{* *}\right)$, ESPCI, 10 rue Vauquelin, \\ 75231 Paris Cedex 05, France \\ $\left({ }^{2}\right)$ Physicochimie Curie $\left({ }^{* * *}\right)$, Institut Curie, 26 rue d'Ulm, 75231 Paris Cedex 05, France
}

(Received 13 March 1997, received in final form 11 April 1997 accepted 6 May 1997)

PACS.61.41.+e - Polymers, elastomers, and plastics

PACS.83.10.Nn - Polymer dynamics

PACS.83.50.By - Transient deformations and flow; time-dependent properties: start-up, stress relaxation, creep, recovery, etc.

\begin{abstract}
We propose a simple scaling argument to describe the propagation of tension along a filament suddenly pulled by one of its ends, a problem recently considered by Seifert, Wintz and Nelson (Phys. Rev. Lett. 77 (1996) 5389-5392).
\end{abstract}

\section{Introduction}

Seifert, Wintz and Nelson (SWN) [1] have recently analyzed the propagation of a strong tension along a filament of length $L$ when a force $f$ is suddenly applied to one of its ends, the other end being fixed. The filament is initially almost straight with slight undulations described by equilibrium statistics with a Hamiltonian of the form $\left(k_{\mathrm{B}} T / 2\right) \int \mathrm{d} q A^{2 b-3} q^{2 b} \hat{u}(q) \hat{u}(-q)$, where $\hat{u}(q)$ is the Fourier transform of the displacement $u(x)$ perpendicular to a reference axis $x$. The problem is thus different from the unwinding of a coily flexible polymer [2]. Cases of usual interest are semi-flexible filaments $(b=2$ and $A$ is the persistence length, with $L>A$ for the filament to be straight), and strings under a weak initial tension ( $b=1$ and the initial tension is $k_{\mathrm{B}} T / 4$ ). The sudden application of a strong force $f$ could in principle be realized in a controlled manner with modern micro-manipulation techniques [3-6]. To simplify the description of the propagation of this strong tension, SWN neglect thermal fluctuations and the "curvature" energy described by the above quoted Hamiltonian, these two weak elements contributing only in preparing the initial state. Hydrodynamic interactions are also neglected as they are expected to contribute only through logarithms as is usually the case for quasi-linear filaments [7].

The main result of SWN is that, in this intrinsically non-linear problem, the length $\xi(t)$ of chain along which the strong tension has propagated (and thus set into motion) after a time $t$

$\left({ }^{*}\right)$ Author for correspondence (e-mail: armand@turner.pct.espci.fr)

$\left({ }^{* *}\right)$ URA CNRS 1382

$\left({ }^{* * *}\right)$ UMR 168

(C) Les Éditions de Physique 1997 


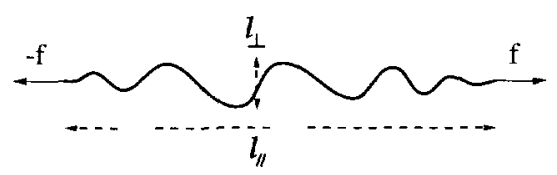

Fig. 1. - A subsection of length $l$ initially displays transverse excursions of order $l_{\perp}$ and a projected length $l_{\|}$. Applying a tension $f$ to the ends induces stretching in a time $t(l)$.

behaves in scaling form as $\xi \sim t^{z}$ with $z \leq 1 / 2$. Two different scaling arguments lead them to two different estimates for the dynamic exponent: $z=(5-2 b) / 4$ and $z=1 / 2$. Their numerical calculations then suggest that $z$ crosses smoothly from the latter to the former as $b$ is increased.

The aim of this Short Communication is to show how this picture can be obtained through a single scaling argument. In addition to providing a derivation of the exponent as a function of $b$, our procedure offers physical insight, in particular as to the relative importance of transverse and longitudinal motions in the dissipation. Our argument is performed in two steps; we have tried to stick to the notation of reference [1].

\section{First Step: Stretching a Subsection}

Take a subsection of length $l$ initially at thermal equilibrium. Its typical transverse excursion $l_{\perp}$ and its projected length $l_{\|}$(see Fig. 1) can be derived from equipartition: $l_{\perp}^{2}=\left\langle u(x)^{2}\right\rangle \simeq$ $\int_{2 \pi / l}^{\Lambda} \mathrm{d} q A^{3-2 b} q^{-2 b}$ and $l_{\|}=l-\delta l$ with $\delta l / l \simeq\left\langle\frac{1}{2}\left(\partial_{x} u\right)^{2}\right\rangle \simeq \int_{2 \pi / l}^{\Lambda} \mathrm{d} q A^{3-2 b} q^{-2 b+2}$ where $\Lambda$ is a microscopic cut-off, and $\delta l / l \ll 1$ is a consistency requirement (so that $l_{\|} \simeq l$ ).

What time $t$ does it take to stretch this subsection by pulling on both its ends with a force $f$ ? This stretching implies transverse displacements of order $l_{\perp}$ and longitudinal ones of order $\delta l$. Thus in scaling form the dissipation balance reads:

$$
f v_{\|} \simeq \Gamma^{-1} l\left(v_{\|}^{2}+v_{\perp}^{2}\right)
$$

where $\Gamma^{-1}$ is the friction coefficient per unit length, and $v_{\|}=\delta l / t$ and $v_{\perp}=l_{\perp} / t$ are the typical extensional and transverse velocities. From (1) we get the stretching time $t$ as a function of $l$ or inversely the maximal length that has been stretched after a time $t: l(t)$.

The dependency on $b$ is quite rich:

- if $2 b \geq 3$ : transverse velocities are larger $\left(v_{\perp} \gg v_{\|}\right)$so

$$
l^{2} \simeq \Gamma f t ; \quad v_{\|} \simeq A^{3-2 b}(\Gamma f t)^{b-1} t^{-1}
$$

- if $3 \geq 2 b \geq 1$ : the situation is more complex: for short subsections $\left(l<l_{\mathrm{c}}=\left(A \Lambda^{2}\right)^{-1}\right)$, $v_{\perp} \gg v_{\|}$, whereas transverse effects are negligible for longer subsections.

Thus, for $l(t) \ll l_{\mathrm{c}}$

$$
l^{2 b-1} \simeq(\Gamma f t) \Lambda^{3-2 b} ; \quad v_{\|} \simeq A^{3-2 b} l^{2 b-2} t^{-1}
$$

whereas for $l(t) \gg l_{c}$,

$$
l^{2} \simeq(\Gamma f t)(A \Lambda)^{2 b-3} ; \quad v_{\| !} \simeq(A \Lambda)^{\frac{3-2 b}{2}}(\Gamma f / t)^{1 / 2}
$$

- if $1 \geq 2 b$ : then transverse effects are always negligible and equation (4) applies. 


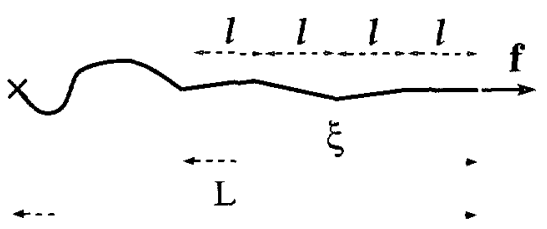

Fig. 2. - The right end of the filament of length $L$ is pulled by a force $f$ at positive times. After a time $t$ a section of length $\xi$ has been set into motion. In the proposed scaling picture, this section can be viewed as a sequence of stretched subsections of length $l(t)$.

\section{Second Step: Pulling the Whole Filament by One End}

Let us return to the initial filament of length $L$, pulled by one end only, the other one being fixed. After a time $t$, a tension of order $f$ has propagated along a section of length $\xi(t)$ which moves at an average velocity $v_{\mathrm{av}}$. At the scaling level, we approximate this section by a simple string of $n(t)=\xi / l$ identical subsections of length $l(t)$ achieving stretching, each extending at a velocity $v_{\|}(l)$ (see Fig. 2). Then, as these extensions accumulate, the average velocity is simply $v_{\mathrm{av}} \simeq n v_{\|}$.

The global force balance on the filament reads:

$$
f=\Gamma^{-1} \xi v_{\mathrm{av}} \simeq \Gamma^{-1} n^{2} l v_{\|}
$$

From equations $(1,5)$ two situations may arise:

i) Locally, longitudinal dissipation dominates $\left(v_{\|} \gg v_{\perp}\right)$, so (1) and (5) coincide with $n=\xi / l=1$ : tension propagation and stretching occur at the same rate.

ii) Locally (in (1)), dissipation is dominated by transverse motions ( $\left.v_{\|} \ll v_{\perp}\right)$. In this case, one gets a solution to (1) and (5) with $n \gg 1$ : tension propagates faster than stretching is achieved. Note: $v_{\mathrm{av}} \simeq v_{\perp}$ so that the stretching of a subsection drifting at $v_{\mathrm{av}}$ is described by the same scaling law $l(t)$ than the stretching of a non-drifting subsection (described by $(1)$ ). This gives consistency to our argument. The global dissipation balance is $f v_{\mathrm{av}} \simeq \Gamma^{-1} \xi\left(v_{\mathrm{av}}^{2}+v_{\perp}^{2}\right)$, so globally, transverse and longitudinal motions contribute comparable amounts.

\section{Conclusion}

So how does the dynamic exponent depend on $b$ ? Estimates for both $\xi$ and the typical velocity of the pulled end $v_{\mathrm{av}}=\Gamma f / \xi$ follow directly from equations (2-5).

If $2 b>3$, then

$$
\xi^{2} \simeq A^{2 b-3}(\Gamma f t)^{\frac{5-2 b}{2}},
$$

in agreement with the adiabatic "Scaling Argument I" in [1]. On the other hand, if $2 b<3$, then

$$
\xi^{2} \simeq(A \Lambda)^{2 b-3}(\Gamma f t)
$$

in agreement with the "Scaling Argument II" in [1]. Note that quite surprisingly this last formula holds for both short and long times for $3>2 b>1$ (i.e. both for $l(t)<l_{\mathrm{c}}$ and $\left.l(t)>l_{\mathrm{c}}\right)$. Thus $z=1 / 2$ is obtained when locally $v_{\|} \gg v_{\perp}$, but not only in that case.

An additional remark: (5) is valid until $\xi=L$ when tension reaches the fixed end. If the chain is stretched at that time $(n=1$ already) nothing more happens. Otherwise, elongation of the chain proceeds with $n(t)=L / l(t)$ and $v_{\mathrm{av}}=n v_{\|}$. For $2 b>3$ this leads to $v_{\mathrm{av}} \simeq(L \Gamma f)(\Gamma f t)^{\frac{2 \mathrm{t}-5}{2}}$, which could be tested in simulations. 
In conclusion, our simple scaling argument allows to recover from a single approach the rich behaviour described by SWN. This argument could be made more precise by the introduction of a tension profile, but we hope that its very simplicity can make it useful to address more complex situations such as the stretching of membranes (as mentioned in [1]), where hydrodynamic interactions should be taken into account.

\section{References}

[1] Seifert U., Wintz W. and Nelson P., Phys. Rev. Lett. 77 (1996) 5389-5392.

[2] Brochard-Wyart F., Hervet H. and Pincus P., Europhys. Lett. 26 (1994) 511-516.

[3] Smith S.B. and Finzi L., Bustamante C., Science 258 (1992) 1122.

[4] Perkins T.T., Smith D.E. and Chu S., Science 264 (1994) 819.

[5] Wirtz D., Phys. Rev. Lett. 75 (1995) 2436-2439.

[6] Cluzel P., Lebrun A., Heller C., Lavery R., Viovy J.-L., Chatenay D. and Caron F., Science 271 (1996) 792.

[7] Farge E. and Maggs A.C., Macromolecules 26 (1993) 5041. 